\title{
FutureJournal
}

\section{Cenários: uma Análise da Produção Científica Brasileira entre 1980 e 2016}

\author{
Leila Aparecida Scherer Weiss \\ Universidade Estadual do Oeste do Paraná (UNIOESTE), Brasil \\ leila_weiss@hotmail.com \\ Jhessica Tamara Kremer \\ Universidade Estadual do Oeste do Paraná (UNIOESTE), Brasil \\ jkarine93@hotmail.com \\ Elizete Tarrago Trentin \\ Universidade Estadual do Oeste do Paraná (UNIOESTE), Brasil \\ elizetetrentin@hotmail.com \\ Claudio Antonio Rojo \\ Universidade Estadual do Oeste do Paraná (UNIOESTE), Brasil \\ rojo_1970@hotmail.com
}

\section{RESUMO}

Este estudo investiga os cenários, sendo desenvolvido com o objetivo de investigar as abordagens sobre cenários nas pesquisas publicadas em periódicos nacionais, abrindo assim o caminho para novas pesquisas. Neste sentido realizou-se uma pesquisa bibliométrica e sociométrica, sem delimitação de tempo, em 84 artigos. A Pesquisa é caracterizada como descritiva quanto aos objetivos, documental quanto aos procedimentos e quantitativa quanto à abordagem do problema. Como resultado, destaca-se a existência de mais pesquisas sobre Cenários Prospectivos e os autores que mais se destacam no cenário nacional são Claudio Antonio Rojo e James Terence Coulter Wright, sendo estes considerados atores centrais em sua rede, tendo em vista o número de laços deste em relação aos outros autores.

PALAVRAS-CHAVE: Cenário. Redes sociais. Bibliometria. 


\section{FutureJournal}

FUTURE STUDIES RESEARCH JOURNAL: TRENDS AND STRATEGIES

\title{
Scenarios: an Analysis of the Brazilian Scientific Production between
} 1980 and 2016

\begin{abstract}
This study investigates the scenarios, being developed with the objective of investigating the approaches on scenarios in the researches published in national newspapers, thus paving the for new bodies of research. A bibliometric and sociometric research was carried out, with no time limitation, in 84 articles. The research is characterized as descriptive regarding the objectives, documentary regarding the proceedings and quantitative regarding the problem approach. As a result, the existence of bodies of research about Prospective Scenarios stands out. The most prominent authors in Brazil are Claudio Antonio Rojo and James Terence Coulter Wright, being considered central authors in their network, considering the number of bonds of these authors in relation to others.
\end{abstract}

KEY-WORDS: Prospective tools. Project risk management. Innovation and technology institutes. 


\section{INTRODUÇÃO}

As inovações organizacionais disseminaram-se para novas práticas empresariais nos procedimentos de tomada de decisão e no cumprimento de suas metas e objetivos. O planejamento estratégico tornou-se uma necessidade na identificação de algumas características e variáveis que influenciam nos critérios adotados em suas filosofias para investimentos de curto e longo prazo, diante ao ambiente competitivo em que se vive. Parafraseando Rojo (2005, p. 22-23), as instituições estão sendo afetadas por essa nova visão de administração.

Segundo Hoss, Bromberger, Rojo e Sousa (2012), "diante das rápidas mutações pelas quais passam as organizações, observa-se que poucas estão preparadas para enfrentar o futuro de maneira sistematizada". Nesse sentido, uma análise nos processos estratégicos adotados por meio de uma simulação dos cenários nas instituições poderá aperfeiçoar o posicionamento de suas ações para estratégicas futuras.

Conforme Rojo e Brandalise (2010), a decisão estratégica nas empresas é influenciada por diversas variáveis e a construção de uma simulação de cenários poderá otimizá-la. Ao simular cenários, as organizações ampliam sua capacidade de visualizar suas ações estratégicas para o futuro, como forma de atingir seus objetivos (Hoss et al., 2012).

A simulação de cenários é como trazer o futuro para uma análise presente, antecedendo o ato de planejar na redução do elemento surpresa no âmbito dos negócios. As decisões do presente são embasadas em antecipações de futuros prováveis nas imagens dos cenários, as incertezas em relação ao futuro não são eliminadas por meio dos cenários, mas poderão ser reduzidas, desde que organizadas e limitadas em números de alternativas com maior probabilidade de ocorrer, a flexibilidade e atualização da simulação dos cenários é de vital importância na identificação dos acontecimentos futuros (Rojo, 2006).

Para Rojo (2010), os dados e as opiniões qualitativas apresentadas nos cenários oferecem possibilidade de previsão dos acontecimentos futuros. Com o estudo de um cenário qualitativo que explore acontecimentos futuros que possam afetar a rentabilidade nas empresas, é possível construir 
antecipadamente elementos para a formação de estratégias em um ambiente organizacional, oferecendo suporte na tomada de decisão (Tadeu \& Silva, 2013).

Campos (1998) remete à ideia de que, ao medir, o ser humano entende; ao entender, conhece; e conhecendo, tem o poder. Assim o conjunto de possibilidades criado por meio dos resultados obtidos dos cenários pesquisados em uma sequência de eventos, expandem o pensamento do ser humano, implementando decisões do passado, limitando os riscos e os erros que podem ocorrer no processo da tomada de decisões, otimizando resultados (Tadeu \& Silva, 2013).

Nesse sentido, para se conquistarem novas oportunidades no mercado, devem-se adotar estratégias adequadas a suas capacitações e utilizá-las da melhor forma possível (Hoss et al., 2012). Diante desse fato, Tadeu e Silva (2013, p. 6) destacam que "o desenvolvimento de cenários permite o aprofundamento nos fatores geradores das mudanças".

Perante o exposto, surge a seguinte questão de pesquisa: Quais abordagens sobre Cenários são encontradas nas pesquisas publicadas em periódicos nacionais? Dessa forma, no presente trabalho, o objetivo foi investigar as abordagens sobre cenários no contexto das pesquisas publicadas em periódicos nacionais, publicados a partir do ano de 1980, uma vez que não foram encontrados estudos em anos anteriores que tenham sido publicados nas bases utilizadas na pesquisa.

A despeito da importância do desenvolvimento de cenários para o desenvolvimento das organizações e ainda que tenham sido realizados outros estudos sobre a produção científica na área de administração e estratégias, não se identificaram, até o momento, estudos que tenham investigado abordagens e redes de relacionamentos nesta área.

Portanto, o trabalho justifica-se pela importância do tema pesquisado como ferramenta estratégica emergencial no ambiente organizacional, no enfrentamento de fatores concorrenciais e nas inovações que de alguma forma venham a surgir, necessitando, desse modo, de mais estudos sobre o assunto e abrindo, assim, caminho para novas pesquisas. 
Nesse sentido, a principal contribuição pretendida pelo estudo é apresentar como as pesquisas vêm sendo publicadas e criar vínculo para futuras pesquisas sobre o tema, avaliando e discutindo como se deu a propagação dos estudos sobre cenários e como esta área se consolidou como novo modelo de gestão no Brasil. O artigo encontra-se estruturado da seguinte forma: Introdução, Fundamentação Teórica, Metodologia, Discussão e Análise dos Dados e Considerações Finais.

\section{FUNDAMENTAÇÃO TEÓRICA}

Ao serem inseridas no mercado, as empresas sofrem com a alta concorrência, que não se manifesta apenas nos concorrentes, mas também na subjacente economia e forças competitivas (Porter, 1979). Assim para enfrentarem a concorrência e se manterem vivas no mercado, as organizações precisam antever o mercado, formulando cenários estratégicos e, por consequente, seu planejamento empresarial.

Em termos históricos, o planejamento empresarial, de forma geral, tomava embasamento nos julgamentos oriundos da experiência e intuição profissional, no entanto, devido à elevada especialização e aos avanços tecnológicos dos sistemas de produção, foi necessário o desenvolvimento formal de técnicas de planejamento baseadas na construção e análise de modelos matemáticos para a tomada de decisão das empresas (Tadeu \& Silva, 2013).

Tomar decisões é uma ação corriqueira para os administradores, que, no entanto, deve estar relacionada à avaliação das alternativas, sendo necessário que todas atendam a um conjunto de objetivos pretendidos, mas a questão problema é: qual a alternativa que melhor atende ao conjunto de objetivos pretendidos? (Tadeu \& Silva, 2013).

Para Rodrigues, Rojo e Bertolini (2013), em um mercado atual repleto de incertezas, os indivíduos necessitam ter o potencial de indagar os pressupostos da maneira como o mercado funciona, buscando ter uma visão mais clara sobre ele. Nesse contexto, surgem os cenários, os quais possuem o objetivo de auxiliar na mudança de visão sobre a realidade, buscando aproximar essa visão da realidade de como ela é e como será. Ao planejar 
cenários, assume-se uma incerteza, fato que, ao ser inserido na cultura organizacional, irá colocar nas mãos dos gestores um diferencial estratégico, reduzindo riscos na tomada de decisão e elevando a probabilidade de acerto, ao levar em consideração tais mudanças inevitáveis, permitindo que a empresa caminhe por um percurso com chances de maior assertividade em sua sobrevivência, visto que oferta melhores condições de competitividade e adaptação (Rodrigues et al., 2013).

Prever os acontecimentos e olhar para o futuro, portanto, auxilia as empresas a tomar suas decisões e dizimar as incertezas existentes no caminho das empresas, auxiliando, portanto, na definição e projeção de situações futuras e favorecendo o planejamento das ações dos gestores organizacionais (Barbosa \& Gatti, 2012).

Segundo Rojo (2005, p. 99), "em uma perspectiva organizacional ampla, cenários são possibilidades de acontecimentos futuros, que, se parametrizadas, podem ser simuladas" e envolvem algumas percepções diferentes, conforme visto no Quadro 1, a seguir.

\begin{tabular}{|c|c|}
\hline No. & Percepção \\
\hline 1 & Nenhum cenário é exato, pois apenas prevê um futuro e não o cria. \\
\hline 2 & $\begin{array}{l}\text { Planejar cenários permite explorar resultados possíveis em várias alternativas } \\
\text { de futuro. }\end{array}$ \\
\hline 3 & $\begin{array}{l}\text { A análise por cenários permite alcançar um nível mais elevado de } \\
\text { aprendizagem organizacional. }\end{array}$ \\
\hline 4 & $\begin{array}{l}\text { Executivos envolvidos na análise por cenários permite alavancar a } \\
\text { prosperidade da empresa através da aprendizagem em equipe. }\end{array}$ \\
\hline 5 & $\begin{array}{l}\text { O planejamento estratégico, formulado com base nos cenários, é uma } \\
\text { experiência dinâmica, vital e compartilhada para os executivos. }\end{array}$ \\
\hline 6 & $\begin{array}{l}\text { A análise por cenários permite buscar saídas antecipadas para o que pode vir } \\
\text { a acontecer. }\end{array}$ \\
\hline 7 & $\begin{array}{l}\text { Financeiramente, a análise por cenários é mais focada em resultados } \\
\text { mensuráveis do que nas demais variáveis de um mercado competitivo. }\end{array}$ \\
\hline 8 & $\begin{array}{l}\text { Para moldar uma análise por cenários, pode ser necessário que ser faça uma } \\
\text { combinação de critérios baseados em determinadas preferências situacionais. }\end{array}$ \\
\hline 9 & $\begin{array}{l}\text { Antever os acontecimentos é jogar com as possibilidades de êxito, analisando } \\
\text { os riscos e decidindo com base nas variáveis que compõem o ambiente. }\end{array}$ \\
\hline 10 & $\begin{array}{l}\text { O grau de complexidade do processo de simulação de cenários não deve ser } \\
\text { subestimado por seus executores. }\end{array}$ \\
\hline 11 & $\begin{array}{l}\text { Maneiras de acompanhar as mutações dos cenários são flexibilizando e dando } \\
\text { continuidade à abertura de aprendizado. }\end{array}$ \\
\hline 12 & $\begin{array}{l}\text { A limitação do número de cenários é necessária para a etapa posterior, o } \\
\text { planejamento estratégico. }\end{array}$ \\
\hline 13 & $\begin{array}{l}\text { É necessário que haja comprometimento dos gestores para com a análise por } \\
\text { cenários. }\end{array}$ \\
\hline 14 & $\begin{array}{l}\text { A participação de colaboradores táticos e funcionais é importante no } \\
\text { diagnóstico, montagem e execução dos cenários. }\end{array}$ \\
\hline
\end{tabular}




\section{Quadro 1: Percepções sobre cenário}

Fonte: Rojo (2005)

Como destacado por Rojo (2005), a utilização de cenários como metodologia de negócio, deve levar em consideração que o cenário não é exato, uma vez que ele apenas projeta situações que poderão ou não acontecer no futuro, podendo existir uma diversidade de resultados, assim como possibilita uma variedade de resultados planejados.

Cenários também podem ser úteis para os gestores, pois, ao permitir a criação de possíveis situações futuras, aumentam a percepção dos gestores quanto a mudanças do ambiente, elevando o nível de conhecimento dos administradores, assim como estimulam o aprendizado, enriquecendo o debate acerca de pontos críticos sobre o futuro das organizações, promovendo o desenvolvimento e análise de novas opções de futuro, com maior transparência e por todos os que fazem parte da empresa (Costa, Fischmann, Boaventura, Muniz \& Nery, 2007).

Com o propósito de auxiliar os gestores a compreender as possíveis repercussões e desdobramentos do ambiente econômico, surgem os conhecidos cenários estratégicos, ferramentas que orientam empresas e governos, como uma referência para examinar alternativas e tomadas de decisões ao encontro de um objetivo estratégico (Schwartz, 1991).

Por mais que almejem, em alguns casos, diferentes horizontes, o objetivo dos cenários é a formulação de opções de longo prazo, buscando contribuir na formação de uma visão compartilhada de negócios e objetivos a serem alcançados, considerando as dificuldades e as restrições de uma ótica sobre o curto prazo (Schwartz, 1991).

O planejamento por cenários é utilizado também para identificar possíveis oportunidades de negócios, testar determinada estratégia em diversos cenários, fazer o monitoramento da execução da estratégia, pesquisar alterações no ambiente para formular estratégias que serão adaptadas e/ou alteradas para manutenção das atividades, redução de incertezas com relação à capacidade da liderança de promoção de ajustes, promoção da percepção e geração de novas oportunidades, complementação 
na qualidade do pensamento estratégico e redução de um comportamento rotineiro, operacional ou burocrático (Ribeiro, 2006).

Dessa maneira, o planejamento tendo como base a criação de cenários deve ser considerado como uma ferramenta utilizada pelas organizações para antever possíveis futuros e construir memórias, preparando-se para o futuro (Blois, Padilha, Medeiros, Cruz, \& Castilhos Spanholi, 2015). No entanto, para Ribeiro (2006), planejar por meio de cenários requer que sejam seguidos passos sistematizados e identificáveis, processo que requer alta interação e imaginação. O primeiro passo é o isolamento da decisão a ser tomada, desafiando rigorosamente os mapas mentais que formam a percepção do individuo e fazem com que se pesquisem e construam informações de fontes pouco ortodoxas. Os passos seguintes são mais analíticos:

- identificar as forças motrizes (sociais, tecnológicas, ambientais, econômicas e políticas);

- identificar os elementos predeterminados;

- identificar o que é inevitável (como aspectos demográficos que já estão em curso) e as incertezas críticas;

- identificar o que é imprevisível ou uma questão de escolha (como a opinião pública).

Tais fatores são, então, priorizados de acordo com sua importância e incerteza. Essa atitude resulta na elaboração cuidadosa de três ou quatro cenários (Ribeiro, 2006).

A metodologia para a construção de cenários a fim de chegar-se ao resultado de um cenário segue a seguinte ordem cronológica: 1) preparação de estudo, 2) diagnóstico da situação atual, 3) determinação das variáveis relevantes, 4) consenso sobre as variáveis relevantes, 5) análise das mútuas influências entre as variáveis relevantes.

Quanto à referência às formas básicas de desenvolvimento de cenários, os executivos da empresa podem considerar duas situações principais: abordagem projetiva e abordagem prospectiva.

A abordagem projetiva é conhecida por restringir-se a fatores e variáveis quantitativos, objetivos e conhecidos, buscando explicar o futuro 
pelo passado, considerando o futuro como único e certo, e utilizando modelos determinísticos e quantitativos (Oliveira, 2001).

Pode-se dizer que a abordagem projetiva faz um cálculo antecipado de uma situação futura com base em dados parciais insuficientes para direcionar adequadamente a ação da empresa no futuro.

A abordagem prospectiva considera outros aspectos, como visão global; variações qualitativas, quantificáveis ou não, subjetivas ou não, conhecidas ou não; ocorrência de um futuro múltiplo e incerto; futuro como determinante da ação presente; e uma análise com intenção, em que os gestores podem fazer uso de variáveis de opinião, as quais são analisadas por métodos do tipo estrutural, impactos cruzados, como a Técnica Delphi (Teixeira, 2005).

Pode-se dizer que a abordagem prospectiva é o conjunto de pesquisas a respeito de fenômenos técnicos, tecnológicos, científicos, econômicos, sociais, entre outros, que objetiva prever a evolução futura das sociedades e cria futuros desejáveis e viáveis.

No que tange ao desenvolvimento de cenários, os gestores da entidade podem considerar alguns módulos de ação interatuantes, são eles: módulo tecnológico, módulo político-econômico, módulo produto e prestação de serviços, módulo propósitos atuais e potenciais e módulo sociocultural.

O módulo tecnológico aborda as principais tendências tecnológicas, os potenciais de desenvolvimento de novas aplicações e as respectivas capacitações necessárias; o módulo político-econômico estuda as projeções dos objetivos da realidade da macro e da microeconomia, traduzidas nas pressões externa e interna, direta e/ou indiretamente agindo sobre o setor de atuação da empresa; o módulo produto e prestação de serviços analisa as tendências do conjunto de produtos e serviços oferecidos no que se refere a seus múltiplos aspectos de capacitação em infraestrutura e de operação; o módulo propósitos atuais e potenciais estuda a situação futura dos vários segmentos em que a empresa atua e/ou em que existe a possibilidade de algum dia vir a atuar; e o módulo sociocultural estuda as evoluções do quadro social e cultural, principalmente quanto aos valores que apresentam maior interação com os outros módulos (Matos, Matos \& Almeida, 2007). 
Os cenários podem ser classificados em realista, pessimista e otimista. O pessimista projeta situações desfavoráveis para a empresa, as quais são demonstradas no planejamento estratégico, além de demonstrar as estratégias e as ações para superar tais situações que afetem o desenvolvimento organizacional. O realista projeta situações dentro de um universo, procurando demonstrar uma visão do que provavelmente possa vir a acontecer. O otimista projeta situações ideais para a empresa, não havendo grandes dificuldades para que a organização atinja seus objetivos, e as estratégias procuram manter uma situação de conforto, crescimento e competitividade.

\section{METODOLOGIA}

Quanto à metodologia empregada neste estudo, referentemente aos objetivos, é descritiva; quanto aos procedimentos, trata-se de uma pesquisa documental; e, no que tange à abordagem do problema, é quantitativa (Raupp \& Beuren, 2009). Com o objetivo de alcançar os objetivos desta pesquisa, fez-se uso de análise bibliométrica e sociométrica.

A bibliometria é um dos métodos mais utilizados na avaliação da produção em determinada área, com o propósito de saber sobre sua evolução, em que estágio se encontra e quais as tendência esperadas (Dantas, Silva, Santana \& Vieira, 2011). A bibliometria desenvolveu um grupo de leis que permite a organização dos estudos de forma sistemática: 1) Lei de Lotka, que mede a produtividade dos autores; 2) Lei de Bradford, que mede o grau de atração dos periódicos sobre determinado assunto; e 3) Lei de Zipf, que incide na medição de frequência das palavras em vários textos (Machado, Souza, Palmisano, Campanário \& Parisoto, 2014; Moretti \& Campanario, 2009).

A sociometria volta-se para a exploração da matriz de relacionamentos, a qual se estabelece entre os atores sociais (Wasserman \& Faust, 1994). As relações sociais entre autores criam laços, que, sendo fortes, demonstram a identidade existente entre os indivíduos; já interações com laços fracos possibilitam a conexão entre grupos (Granovetter, 1983). O autor ressalta que laços são essenciais para desenvolvimento, disseminação e 
inovação do conhecimento, pois formam redes de relacionamento constituídas por pesquisadores com experiências e formações distintas. Segundo Wasserman e Faust (1994), as redes sociais podem ser formadas por dois elementos - atores (pessoas, instituições ou grupos) e suas conexões.

O universo desta pesquisa é composto por publicações nacionais nas fontes de dados: Scientific Periodicals Electronic Library (Spell) e Portal de Periódicos CAPES/MEC. A opção por essas duas bases de dados descritas deve-se a sua importância e representatividade no contexto brasileiro.

Os artigos objeto da análise foram obtidos, sem delimitação de tempo, sendo coletados 168 artigos que possuíssem em suas palavras-chave as expressões "cenários" e "cenário", uma vez que o foco da pesquisa baseiase em estudos sobre esse tema. Os artigos localizados passaram por um processo de revisão dos conteúdos para sua classificação em dois grupos: artigos que continham cenários em seus conteúdos ou que aplicavam a técnica de cenários em alguma perspectiva organizacional, e artigos que apenas citavam cenários, ou os utilizavam em outros contextos. Os artigos enquadrados no segundo grupo foram excluídos da pesquisa, pois não faziam parte do objetivo da pesquisa, restando apenas 84 publicações, em diferentes periódicos nacionais.

A análise dos dados foi realizada referentemente a período de publicação dos artigos, periódico, rede social contendo os autores mais prolíficos e com maior número de laços, abordagens dos artigos. Foi utilizada análise das redes sociais, as quais, segundo Matheus e Silva (2006), estudam as ligações relacionais (da expressão em inglês relational tie) entre atores sociais, sendo de interesse a pesquisadores de vários campos do conhecimento que, buscando entender seu impacto sobre a vida social, deram origem a diversas metodologias de análise que têm como base as relações entre os indivíduos, numa estrutura em forma de redes.

Com relação à análise das redes sociais, foi escolhida a exploração das redes de coautoria, o que representa uma vertente de análise de redes sociais (Liu, Bollen, Nelson \& Van de Sompel, 2005). Na geração das figuras representativas da estrutura da rede e seus indicadores, fez-se uso do software Package For Social Network Analysis and Cultural Domain Analysis 
(UCINET\& 6). A busca pela identificação das abordagens dos artigos foi feita por meio de análise de conteúdo temática do objetivo e do resumo dos artigos. A limitação deste estudo encontra-se nos termos de busca "cenário" e "cenários", além das fontes de dados: Scientific Periodicals Electronic Library (Spell) e Portal de Periódicos CAPES/MEC.

\section{DISCUSSÃO E ANÁLISE DOS DADOS}

Nesta seção, procede-se à apresentação, análise e interpretação dos resultados obtidos

Os artigos objetivos do presente estudo foram obtidos sem recorte de tempo, sendo analisados 84 artigos científicos, publicados em diferentes fontes de dados. Foram registradas 45 revistas que abordaram os tema, sendo listadas as com maior número de publicações, as demais, que contavam com apenas um artigo sobre o tema, foram agrupadas na categoria "outros", conforme destacado na Tabela 1.

Tabela 1: Fonte de dados da pesquisa

\begin{tabular}{|c|c|c|c|c|c|c|c|c|c|c|c|c|c|c|c|c|}
\hline Revista & 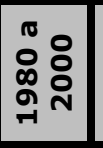 & 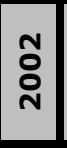 & 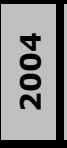 & น̊ & $\hat{\text { ¿ }}$ & $\begin{array}{l}\infty \\
\stackrel{0}{ } \\
\text { ¿े }\end{array}$ & 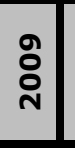 & 울 & ت. & స̃. & $\stackrel{m}{\stackrel{m}{N}}$ & $\begin{array}{l}\text { गे } \\
\text { ते }\end{array}$ & ำ & $\begin{array}{l}0 \\
\stackrel{1}{0} \\
\text { N }\end{array}$ & Total & Frequência \\
\hline Future S. R. J. & & & & & & & 1 & 3 & 2 & 3 & 2 & 1 & & 1 & 13 & $15.48 \%$ \\
\hline RAUSP & 5 & & & & 1 & & & & 1 & & 2 & 1 & & & 10 & $11.90 \%$ \\
\hline RAE & 1 & 1 & & & & 1 & & & & & & & & & 3 & $3.57 \%$ \\
\hline Ibero-Ame. & & & & & & & & 1 & & 2 & & & & & 3 & $3.57 \%$ \\
\hline RCA. & & & & 1 & & & & & 1 & 1 & & & & & 3 & $3.57 \%$ \\
\hline ReCA. & & & 1 & & & & & & & & 1 & & & & 2 & $2.38 \%$ \\
\hline RAP & 2 & & & & & & & & & & & & & & 2 & $2.38 \%$ \\
\hline $\mathrm{G} \& \mathrm{~T}$ & & & & & & 2 & & & & & & & & & 2 & $2.38 \%$ \\
\hline RAI & & & & & & & 1 & & & & & 1 & & & 2 & $2.38 \%$ \\
\hline Turismo V. e A & & & & & 1 & & & & & & & 1 & & & 2 & $2.38 \%$ \\
\hline Cad. V. Turismo & & & 1 & & 1 & & & & & & & & & & 2 & $2.38 \%$ \\
\hline EBAPE & & & & & & & 1 & & & 1 & & & & & 2 & $2.38 \%$ \\
\hline Revista Alcance & & & 1 & & & & & & 1 & & & & & & 2 & $2.38 \%$ \\
\hline RBTur & & & & & & 1 & 1 & & & & & & & & 2 & $2.38 \%$ \\
\hline FACES & & & & & & 2 & & & & & & & & & 2 & $2.38 \%$ \\
\hline
\end{tabular}




\begin{tabular}{l|r|r|r|r|r|r|r|r|r|r|r|r|r|r|r|r}
\hline RTA & & & & & & & 1 & & & 1 & & & & & 2 & $2.38 \%$ \\
\hline REUNA & & & & & & & & & 1 & 1 & & & & & 2 & $2.38 \%$ \\
\hline Outros & 0 & 0 & 0 & 1 & 0 & 2 & 2 & 3 & 2 & 3 & 5 & 2 & 4 & 2 & 28 & $33.33 \%$ \\
\hline Total & $\mathbf{8}$ & $\mathbf{1}$ & $\mathbf{3}$ & $\mathbf{2}$ & $\mathbf{3}$ & $\mathbf{8}$ & $\mathbf{7}$ & $\mathbf{7}$ & $\mathbf{8}$ & $\mathbf{1 2}$ & $\mathbf{1 0}$ & $\mathbf{6}$ & $\mathbf{4}$ & $\mathbf{3}$ & $\mathbf{8 4}$ & $\mathbf{1 0 0 \%}$ \\
\hline
\end{tabular}

Fonte: Dados da Pesquisa

A não delimitação de tempo para a pesquisa possibilitou relacionar artigos publicados na década de 1980, sendo o primeiro estudo publicado em 1988, na Revista de Administração Pública (RAP). Constata-se que a base com maior número de publicações de artigos sobre cenários foi o periódico Future Studies Research Journal (Future S.R.J.) com 13 artigos publicados, sendo o primeiro no ano de 2009.

Outro periódico que possui um número significativo de publicações sobre o assunto é a Revista de Administração (RAUSP), no entanto, ao contrário do periódico citado anteriormente, esta revista vem publicando artigos sobre cenários ao longo dos anos, cinco foram publicados nos anos anteriores a 2000, um em 1989, dois em 1991, um em 1992 e um em 1993.

A Lei de BradFord possibilita estimar o grau de relevância dos periódicos em determinadas áreas do conhecimento, permitindo afirmar que os periódicos que produzem maiores quantidades de artigos sobre determinado assunto, formam um núcleo que, supostamente, traria maior qualidade e relevância para a área (Guedes \& Borschiver,2005).

Segundo Guedes e Borschiver (2005), outro ponto destacado pela Lei de Bradford é o fato de que os artigos, quando escritos, são submetidos a avaliações por periódicos, e, quando aceitos, são publicados. Assim, quanto mais artigos sobre determinado assunto o periódico publicar, maior será o sucesso do periódico na publicação deste tema, o que se reflete neste estudo, pois, como visto, existe uma procura maior por periódicos com maior quantidade de artigos publicados na área.

Quanto ao número de artigos publicados, observa-se que houve um aumento das publicações nos anos 2008 a 2013, com destaque para o ano 2012, no entanto, nos últimos três anos, houve uma queda considerável em publicações sobre cenários. 
A evolução das pesquisas permite maior compreensão sobre 0 assunto, sendo tal conhecimento necessário para a evolução da ciência, permitindo a identificação de duplicações ou contradições e a determinação de vieses e lacunas de próximos estudos.

Destaca-se que neste estudo não se retrata o universo total de pesquisas realizadas, pois sabe-se de vários trabalhos que têm sido realizados e apresentados em congressos ou mesmo em revistas que não estão indexadas nas duas bases utilizadas.

Conhecer o que já está pesquisado é uma necessidade para o preenchimento de novas lacunas de pesquisa e para a consolidação do conhecimento adquirido, nesse sentido, efetuou-se uma relação dos setores de atuação e abordagens empregados nos estudos, exibida na Tabela 2.

Tabela 2: Setor e Abordagens dos artigos

\begin{tabular}{l|r|r|l|r|r}
\hline \multicolumn{1}{c|}{ Abordagem } & Quant. & \multicolumn{1}{c|}{ A } & \multicolumn{1}{c|}{ Abordagem } & Quant. & \% \\
\hline Cenário em Investimento & 3 & $3.6 \%$ & Cenário no Setor de Turismo & 8 & $9.5 \%$ \\
\hline Cenário no Setor Industrial & 5 & $6.0 \%$ & Cenário Setor Agronegócio & 3 & $3.6 \%$ \\
\hline Técnicas de Cenários & 4 & $4.8 \%$ & Cenário no Setor Moveleiro & 1 & $1.2 \%$ \\
\hline Cenários no Setor Público & 6 & $7.1 \%$ & Cenário Setor Cooperativas & 1 & $1.2 \%$ \\
\hline Cenários no Setor Tecnológico & 6 & $7.1 \%$ & Cenários no Setor Hospitalar & 1 & $1.2 \%$ \\
\hline Cenários Prospectivos & 10 & $11.9 \%$ & Cenário Setor Petroquímico & 3 & $3.6 \%$ \\
\hline Cenário no Setor Calçadista & 1 & $1.2 \%$ & Cenário no Terceiro Setor & 1 & $1.2 \%$ \\
\hline Simulação de Cenários & 4 & $4.8 \%$ & Cenários Insti. Financeiras & 4 & $4.8 \%$ \\
\hline Cenários Temáticos & 1 & $1.2 \%$ & Cenários no Meio Ambiente & 2 & $2.4 \%$ \\
\hline Cenário Setor de Serviços & 1 & $1.2 \%$ & Cenário em Universidades & 2 & $2.4 \%$ \\
\hline Cenário no Setor de Telefonia & 2 & $2.4 \%$ & Administração Estratégica & 3 & $3.6 \%$ \\
\hline Construção/Planejamento Cen. & 6 & $7.1 \%$ & Cenário no Jornalismo & 1 & $1.2 \%$ \\
\hline Cenários Estratégicos & 3 & $3.6 \%$ & Cenário na Construção Civil & 1 & $1.2 \%$ \\
\hline Cenários Setor Recursos Hhídricos & 1 & $1.2 \%$ & & & \\
\hline
\end{tabular}

Fonte: Dados da Pesquisa

Na Tabela 2, clarifica-se a abordagem dos artigos utilizados, sobressaindo a abordagem "cenários prospectivos", identificada em 11,9\% dos artigos analisados, no entanto observa-se uma variedade de abordagens, com estudos desenvolvidos no setor de turismo, no setor público, tecnológico e na construção e planejamento de cenários.

Esse resultado pode indicar que o desenvolvimento de estudos nessa área representa uma oportunidade investigativa aos pesquisadores. Nesse 
sentido, acredita-se que a ampliação de discussões relativas ao desenvolvimento de cenários nos mais amplos segmentos, acabará por contribuir para a construção e manutenção da pesquisa sobre o tema como um todo.

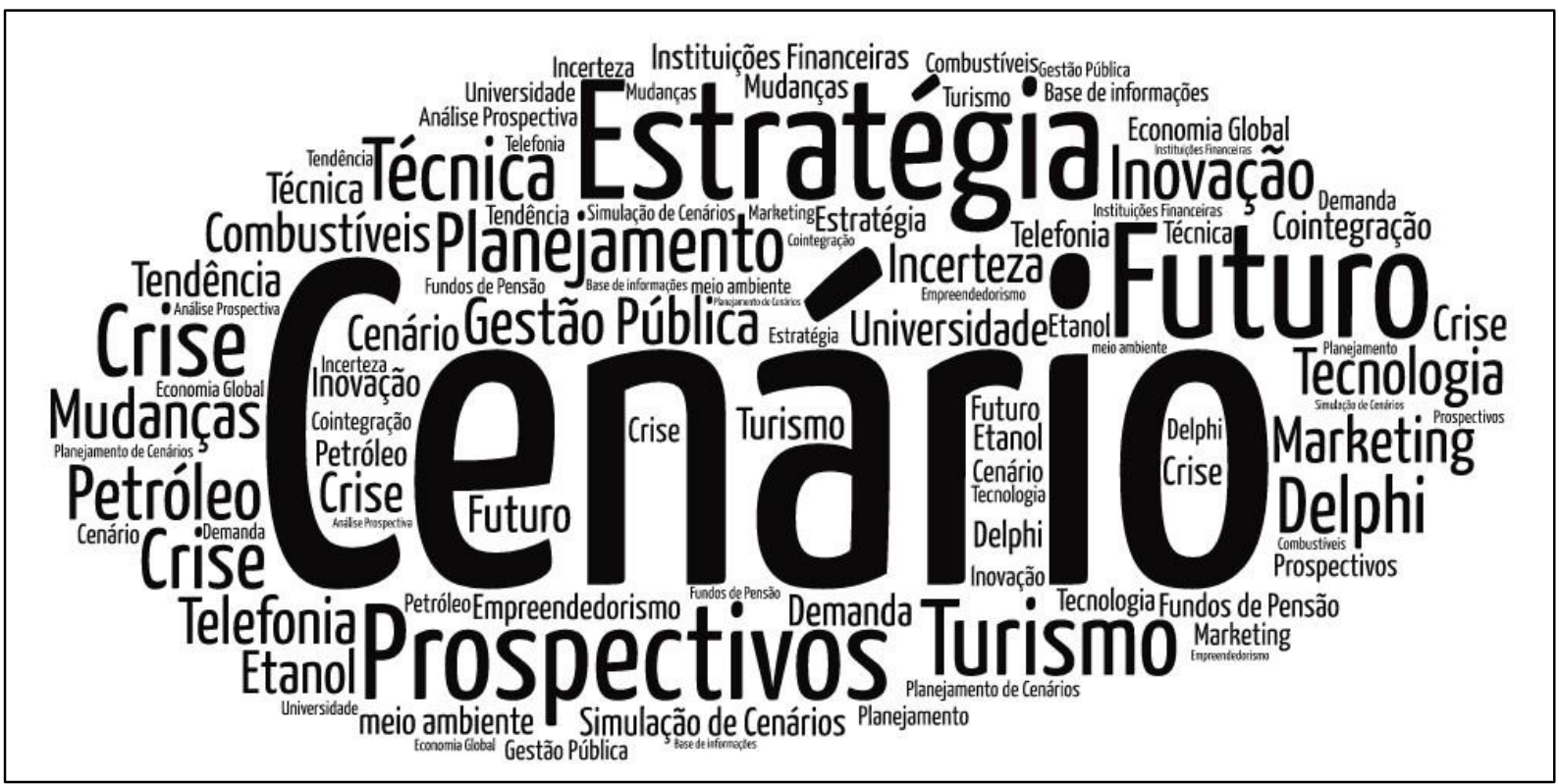

\section{Figura 1: Nuvem de palavras das palavras chave}

Fonte: Dados da Pesquisa

A nuvem de palavras permite que se observem, além da palavra "cenário", os termos "estratégia", "prospectivos", "futuro", "planejamento", que apresentam elevada frequência nas palavras-chave utilizadas nos estudos. Essa elevada frequência dos termos pode ratificar a abordagem dos artigos. A seguir, serão apresentadas algumas das principais abordagens das pesquisas.

Kasznar (1997) projetou um cenário básico para as empresas públicas e privadas para o ano de 1997. Amorim (1997) propôs uma discussão sobre a utilização de cenários de planejamento, para contextualizar as operações planejadas, reduzindo o risco das decisões. 
Costa et al. (2007) investigaram a adequação e a utilização de cenários prospectivos em uma instituição pública do setor de turismo de Natal (RN) e verificou que existe uma lacuna entre a metodologia de cenários e sua aplicação no setor de turismo.

Blois e Souza (2008) propõem uma forma de análise sistêmica do setor calçadista que integre a abordagem de cenários prospectivos e dinâmicas de sistemas, tendo como principal objetivo disponibilizar alternativas que possibilitem a análise, descrição e simulação de tendências de mercado.

Cavalheiro e Fellows (2011) elaboraram uma pequena revisão sobre papel da elaboração de cenários como um dos instrumentos importantes nas análises de futuro, na tentativa de tornar evidente que a função dos cenários não é a de prever o futuro, mas sim a de prever as possibilidades para sua construção.

Brandalise, Rojo, da Mata, Souza (2012) desenvolveram um estudo objetivando realizar uma simulação de cenários e formulação de estratégias competitivas para uma empresa do ramo de confecções, destacando a importância da simulação de cenários para as empresas, para que esta esteja preparada para as mudanças ou acontecimentos do setor.

Hoss et al. (2012) estudaram a metodologia de simulação de cenários, reunindo informações e apresentando os principais conceitos relacionados ao assunto, para aplicá-los nas fontes de recursos da FUNTEFPR, Campus Pato Branco. Apesar de o estudo revelar que raramente um cenário acontecerá exatamente como simulado, ele reduz as incertezas organizacionais vindouras, mostrando-se como uma ferramenta capaz de auxiliar as organizações a definirem suas estratégias e incertezas.

As instituições de ensino são consideradas importantes para a evolução da produção científica, nesse sentido, foram identificadas 50 instituições envolvidas nas publicações analisadas. Para a identificação do vínculo institucional dos autores, utilizaram-se dados constantes nos resumos dos artigos analisados. No caso de autores que indicaram mais de uma instituição, optou-se por considerar somente a primeira instituição informada. 
Na Figura 2, mostra-se a rede completa de interações entre as instituições que se associaram para que os estudos se desenvolvessem. 


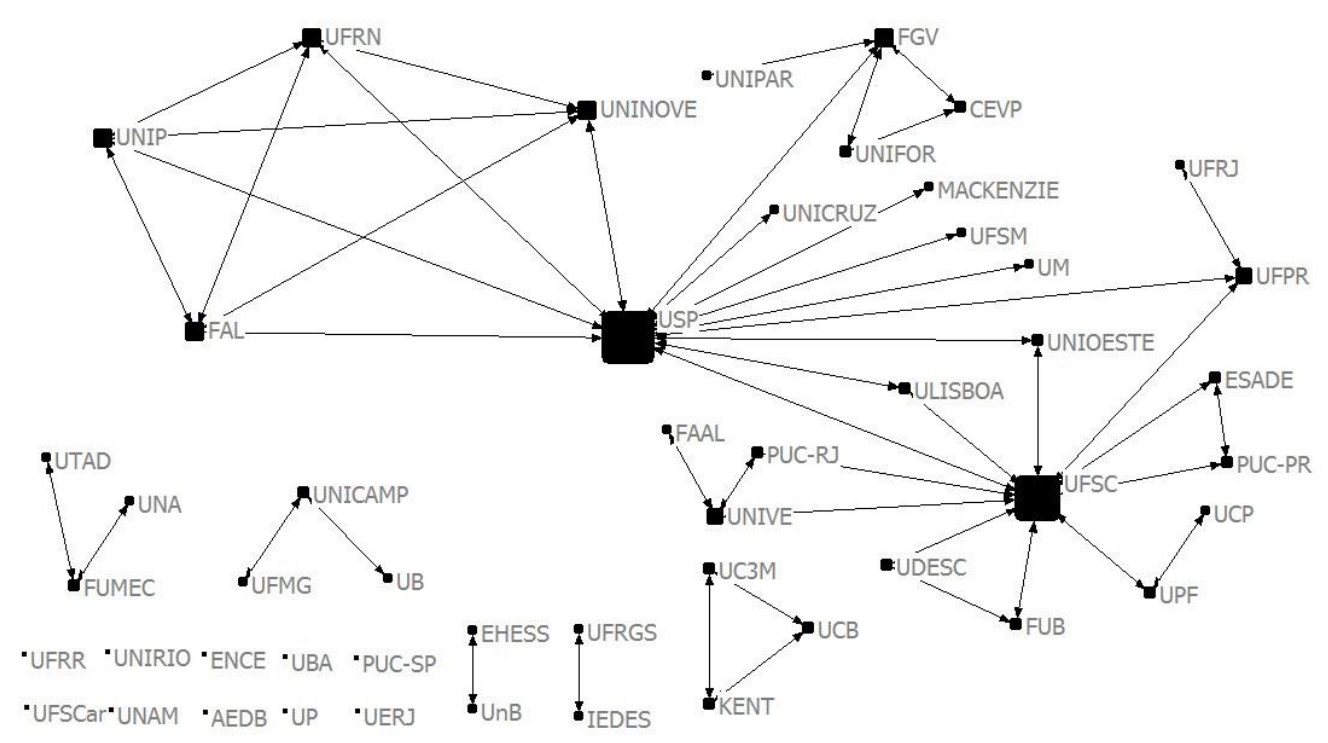

Legenda: Associação Educacional Dom Bosco (AEDB); Centro de Ensino do Vale do Parnaíba (CEPV); Centro Universitário Una (UNA); Centro Universitário Nove de Julho (UNINOVE); Escola Nacional de Ciências Estatísticas (ENCE); Escuela Superior de Administración Y Dirección de Empresas (ESADE); École des Hautes Études en Sciences Sociales de Paris (EHESS); Faculdade de Administração e Artes de Limeira (FAAL); Faculdade de Natal (FAL); Faculdades Unidas de Bagé (FUB); Fundação Getulio Vargas (FGV); Fundação Mineira de Educação e Cultura (FUMEC); Pontifícia Universidade Católica do Paraná (PUC-PR); Pontifícia Universidade Católica de São Paulo (PUC-SP); Pontifícia Universidade Católica do Rio de Janeiro (PUC-RJ); Universidad de Buenos Aires (UBA); Università $\mathrm{Ca}^{\prime}$ Foscari, na Itália (UNIVE); University Carlos III of Madrid (UC3M); University of Kent at Canterbury (KENT); University of Manchester (UM); Universidad de Barcelona (UB); Universidade de Brasília (UnB); Universidade Católica de Brasília (UCB); Universidade Católica de Pelotas (UCP); Universidade de Cruz Alta (UNICRUZ); Universidade de Passo Fundo (UPF); Universidade de São Paulo (USP); Universidade de Trás-os-Montes e Alto Douro (Portugal) (UTAD); Universidade Estadual do Centro-Oeste (UNICENTRO); Universidade Estadual do Oeste do Paraná (UNIOESTE); Universidade Estadual do Rio de janeiro (UERJ); Universidade Estadual de Campinas (UNICAMP); Universidade do Estado de Santa Catarina (UDESC); Universidade Federal do Estado do Rio de Janeiro (UNIRIO); Universidade Federal do Paraná (UFPR); Universidade Federal do Rio de Janeiro (UFRJ); Universidade Federal do Rio Grande do Norte (UFRN); Universidade Federal do Rio Grande Do Sul (UFRGS); Universidade Federal de Santa Maria (UFSM); Universidade Federal de Minas Gerais (UFMG); Universidade Federal de São Carlos (UFSCar); Universidade Federal de Roraima (UFRR); Universidade de Fortaleza (UNIFOR); Universidad Nacional de Misiones (UNAM); Universidade Paranaense (UNIPAR); Université Paris Panthéon-Sorbonne (IEDES); Universidade Paulista (UNIP); Universidade Positivo (UP); Universidade Presbiteriana Mackenzie (MACKENZIE); Universidade Técnica de Lisboa (ULISBOA).

\section{Figura 2: Rede de cooperação entre instituições}

Fonte: Dados da Pesquisa

Na Figura 2, é possível identificar e visualizar nos perfis das instituições de ensino uma diversificação não muito grande, uma vez são poucas as instituições que possuem laços com as demais, grande parte apresenta laços fracos e publicações internas. No entanto, observa-se a existência de uma rede de cooperação envolvendo diversas instituições, na 
qual se destaca a USP em virtude de sua centralidade, em grande parte por esta instituição ser considerada como referência para a formação de pesquisadores.

É possível identificar que a estrutura de relacionamento envolve instituições de outros países, do mesmo modo são encontradas publicações de artigos de autores advindos de instituições estrangeiras, uma característica que remete ao aperfeiçoamento dos profissionais por meio de parcerias com universidades estrangeiras.

Quanto à participação dos autores no campo de cenários e para que fossem respeitadas as diferenciações dos nomes dos autores com a mesma citação nominal, executou-se uma consulta ao Curriculum da Platafoma Lattes, do Conselho Nacional de Desenvolvimento Científico e Tecnológico (CNPq), e após a certeza de se tratarem de autores diferentes, optou-se por manter o sobrenome e citar o primeiro nome por extenso, nos demais casos os nomes permanecem abreviados.

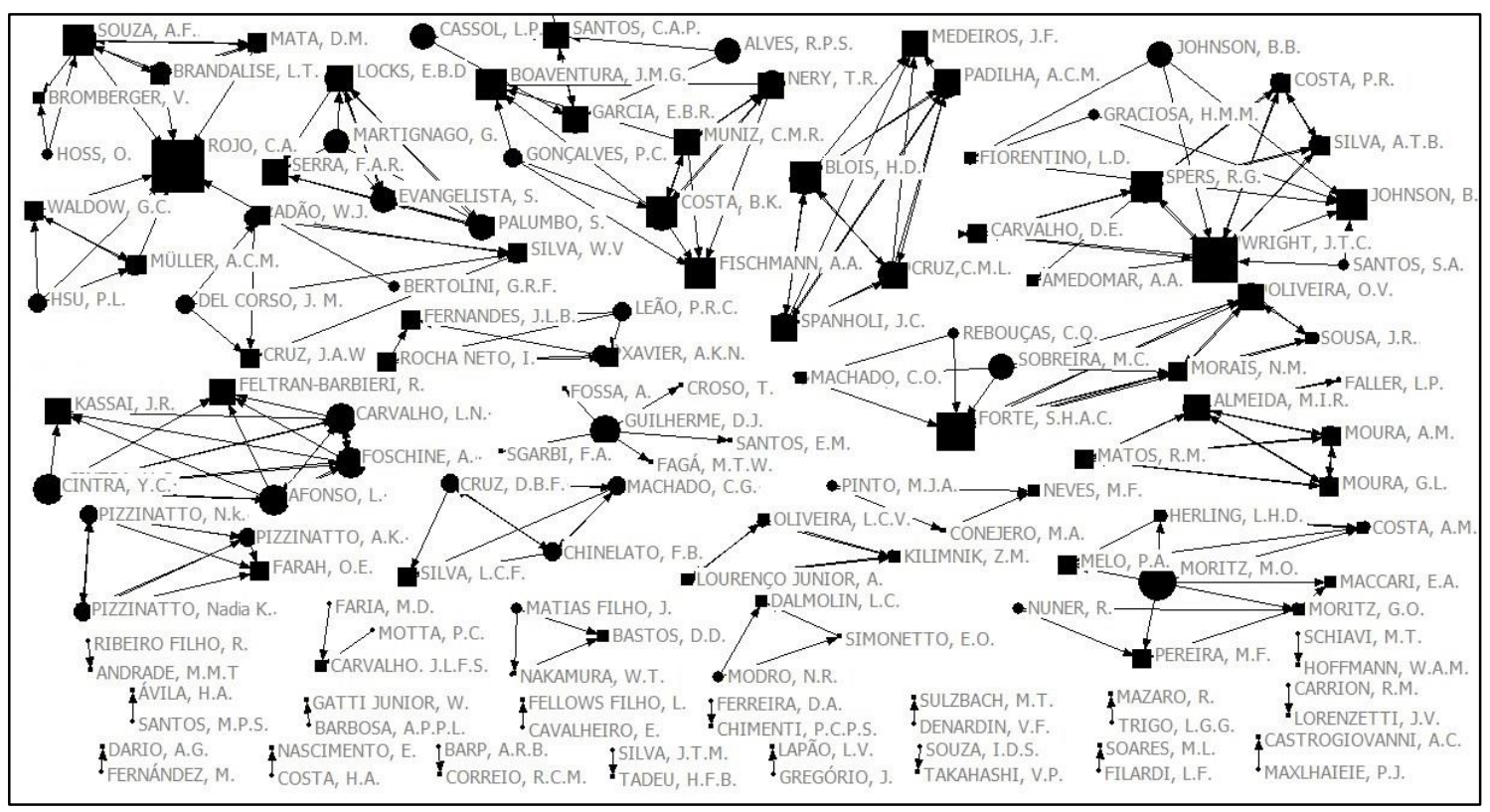

Figura 3: Rede de cooperação entre autores

Fonte: Dados da Pesquisa

Na Figura 3, encontram-se ilustradas as redes de cooperação entre os autores dos artigos analisados, no entanto, para melhor visualização, optou-se por apresentar apenas as redes que envolviam mais de um autor. 
As redes de interação dos atores pode ser considerada como um ambiente de troca e de comunicação que acontece em diversos níveis, em que a quantidade de contatos entre os autores pode ser considerada importante, pois a informação circula entre eles, atingindo todos os atores direta ou indiretamente (Granovetter, 1983).

A análise das redes de cooperação ilustradas na Figura 3 sinaliza que vários autores que se destacam em relação ao número de laços integram a mesma rede de cooperação, como Claudio Antonio Rojo e James Terence Coulter Wright. Observa-se a existência de laços fracos, no entanto são encontrados também laços fortes, que segundo Granovetter (1973) é a conexão direta dos atores em uma rede. Segundo Burt (1992), no contato entre pessoas que já se conhecem, as informações a serem compartilhadas tendem a ser as mesmas, com baixa tendência a mudanças.

Rojo e Wright podem ser considerados atores centrais em sua rede, tendo em vista o número de laços destes em relação aos outros autores, bem como a conexão entre outros grupos de atores. Assim esses dois autores são responsáveis pelos diversos laços, representativos de contatos indiretos formados por meio de pontes, fornecendo diferentes fontes de informação e tornando a rede propensa à inovação (Granovetter, 1973). Da mesma maneira, outros autores formam suas redes de relacionamentos.

\section{CONSIDERAÇÕES FINAIS}

Neste estudo, o objetivo foi investigar as abordagens sobre cenários no contexto das pesquisas publicadas em periódicos nacionais. O estudo justifica-se pela importância do tema pesquisado como ferramenta estratégica emergencial no ambiente organizacional, no enfrentamento de fatores concorrenciais e inovações que de alguma forma venham a surgir, uma vez que, de acordo com Schwartz (1991), cenários são ferramentas que procuram orientar empresas e governos como uma referência para examinar alternativas e tomadas de decisões ao encontro de um objetivo estratégico.

O planejamento por cenários é utilizado também para: a) identificar possíveis oportunidades de negócios; b) testar determinada estratégia em diversos cenários; c) monitor a execução da estratégia adotada; d) pesquisar 
alterações no ambiente para se possa formular estratégias para a manutenção das atividades da empresa; e) reduzir as incertezas sobre a capacidade de liderança; f) promover a percepção e a geração de novas oportunidades; e g) melhorar a qualidade e a percepção a respeito da estratégia de negócios (Ribeiro, 2006).

Foram analisados 84 artigos científicos, publicados em diferentes fontes de dados, sendo o primeiro estudo publicado em 1988, na Revista de Administração Pública (RAP). A análise dos artigos possibilitou estabelecer um panorama das pesquisas realizadas no Brasil desde a década de 1980 e publicados nos periódicos, permitindo com isso, atingir o objetivo proposto.

Constatou-se que o periódico com maior número de publicações de artigos sobre cenários foi o Future Studies Research Journal (Future S.R.J.) com 13 artigos publicados. Verificou-se que houve um aumento das publicações dos anos 2008 a 2013, com destaque para os ano 2012, no entanto nos últimos três anos houve uma queda considerável em publicações sobre o tema.

Quanto à abordagem dos artigos pesquisados, verificou-se a concentração de artigos com a abordagem cenários prospectivos; assim, analisando-se as palavras-chave dos artigos, verificou-se que, além da palavra "cenário", os termos "estratégia", "prospectivos", "futuro", "planejamento" apresentam elevada frequência, o que ratifica a abordagem dos artigos.

As instituições fomentadoras da produção científica nacional detêm grande importância para a disseminação do assunto, observou-se, em se tratando do tema cenários, que as instituições de ensino apresentam uma diversificação não muito grande, uma vez são poucas as que possuem laços com as demais, grande parte apresenta laços fracos e publicações internas. No entanto, observa-se a existência de uma rede de cooperação envolvendo diversas instituições, na qual se destaca a USP em virtude de sua centralidade, em grande parte por esta instituição ser considerada como referência para a formação de pesquisadores. É possível ainda identificar que a estrutura de relacionamento envolve instituições de outros países.

Quanto aos autores, a análise das redes de cooperação sinalizou que autores que se destacam em relação ao número de laços integram a mesma 
rede de cooperação, como Claudio Antonio Rojo e James Terence Coulter Wright, sendo estes considerados atores centrais em sua rede, tendo em vista o número de laços em relação aos outros autores.

O estudo apresenta como limitação o fato de não incorporar no foco de análise publicações sobre o tema fora do âmbito de periódicos nacionais. Sugere-se para pesquisas futuras a utilização análises que envolvam bases de dados internacionais para a seleção de artigos que tratem sobre o tema, além de estudos que foquem os reflexos da construção de cenários em curto e longo prazo.

\section{REFERÊNCIAS}

Amorim, M. C. S. (1997). Planejamento, incerteza e o uso da técnica de cenários. Organizações \& Sociedade, 4(9), 93-101.

Barbosa, A., \& Gatti Júnior, W. (2012). Cenários tecnológicos para a demanda por cana-de-açúcar. Future Studies Research Journal: Trends and Strategy, 4(1), 92-113.

Blois, H. D., \& Souza, J. C. (2008). Cenários prospectivos e a dinâmica de sistemas: proposta de um modelo para o setor calçadista. Revista de Administração de Empresas - RAE, 48(3), 35-45.

Blois, H. D., Padilha, A. C. M., Medeiros, J. F. de, Cruz, C. M. L., \& Castilhos Spanholi, J. d. (2015). A aplicação da ferramenta de cenários prospectivos em um bairro de baixa renda localizado em Passo Fundo, Rio Grande do Sul. Revista de Administração da UFSM, 8(4), 580-597.

Brandalise, L. T., Rojo, C. A., da Mata, D. M., \& de Souza, A. F. (2012). Simulação de cenários e formulação de estratégias competitivas: o caso do atacado liderança. Revista Gestão \& Tecnologia, 12(3), 223-257.

Burt, R. S. (1992). Structural hole. Cambridge, MA: Harvard Business School Press.

Campos, J. A. (1998). Cenário balanceado: painel de indicadores para a gestão estratégica dos negócios. São Paulo: Aquariana.

Cavalheiro, E., Fellows Filho, L. (2011). Strategic scenarios: an aplication in the biotechnology/Cenários estratégicos: uma aplicação na área de biotecnologica. Future Studies Research Journal: Trends and Strategy, $3(1), 2-14$.

Costa, B. K., Fischmann, A. A., Boaventura, J. M. G., Muniz, C. M. R., \& Nery, T. R. (2007). Adequação e uso de cenários prospectivos: um estudo no órgão municipal de turismo de Natal. Turismo-Visão e Ação, 9(1), 7-18. 
Dantas, J. A., Silva, C. A. T., Santana, C. M., \& Vieira, E. T. (2011). Padrões de comunicação científica em contabilidade: um comparativo entre a Revista Contabilidade e Finanças e a The Accounting Review. Revista Contemporânea de Contabilidade, 8(16), 11-36.

Guedes, V. L., \& Borschiver, S. (2005). Bibliometria: uma ferramenta estatística para a gestão da informação e do conhecimento, em sistemas de informação, de comunicação e de avaliação científica e tecnológica. Anais do Encontro Nacional de Ciência da Informação, 6, Salvador, BA, Brasil.

Granovetter, M. S. (1973). The strength of weak ties. American Journal of Sociology, 78(6), 1360-1380.

Granovetter, M. (1983). The strength of weak ties: a network theory revisited. Sociological Theory, (1), 201-233.

Hoss, O., Bromberger, V., Rojo, C. A., \& de Sousa, A. F. (2012). Simulação de cenários: estudo de caso nas fontes de recursos da Fundação de Apoio à Educação, Pesquisa e Desenvolvimento Científico e Tecnológico da Universidade Tecnológica Federal do Paraná no Câmpus Pato Branco. Revista Ibero-Americana de Estratégia, 11(3), 172-204.

Kasznar, I. K. (1997). Produção e desenvolvimento nos estados e municípios. Revista Brasileira de Administração Pública - RAP., 31, 223-254.

Liu, X., Bollen, J., Nelson, M. L., \& Van de Sompel, H. (2005). Co-authorship networks in the digital library research community. Information Processing \& Management, 41(6), 1462-1480.

Machado Junior, C., Souza, M. T. S., Palmisano, A., Campanário, M. A., Parisotto, I. R. S. (2014). Análise de viabilidade de utilizar as leis da bibliometria em diferentes bases de pesquisa. Anais do Encontro Anual da Associação de Pós-Graduação e Pesquisa em Administração, 38, Rio de Janeiro, RJ, Brasil.

Matheus, R. F., \& Silva, A. B. D. O. (2006). Análise de redes sociais como método para a Ciência da Informação. DataGramaZero-Revista de Ciência da Informação, 7(2).

Matos, J. G. R., Matos, R. M. B., \& de Almeida, J. R. (2007). Análise do ambiente corporativo: do caos organizado ao planejamento estratégico das organizações. Rio de Janeiro: E-papers.

Moretti, S. L. A., \& Campanário, M. A. (2009). A produção intelectual brasileira em Responsabilidade Social Empresarial-RSE sob a ótica da bibliometria. Revista de Administração Contemporânea - RAC, 13(edição especial), 68-86.

Oliveira, D. P. R. (2001) Planejamento Estratégico. Conceitos. Metodologia. Práticas. 16a ed. São Paulo: Atlas. 
Porter, M. E. (1979, March-April). How competitive forces shape strategy. Havard Business Review, 57(2), 137-145.

Raupp, F. M; Beuren, I. M. (2009). Metodologia da pesquisa aplicável às ciências sociais. In I. M. Beuren (Org.), Como elaborar trabalhos monográficos em contabilidade: teoria e prática (3a ed). São Paulo: Atlas.

Ribeiro, M. D. P. M. (2006). Planejamento por cenários: uma ferramenta para a era do conhecimento. Revista Intersaberes, 1(1), 186-202.

Rodrigues, A., Rojo, C. A., \& Bertolini, G. R. F. (2013). Formulação de estratégias competitivas por meio de análise de cenários na construção civil. Produção, 23(2), 269-282.

Rojo, C. A. (2005). Modelo para a simulação de cenários: uma aplicação em instituição de ensino superior privada. Tese de Doutorado, Universidade Federal de Santa Catarina: Florianópolis, SC, Brasil.

Rojo, C. A. (2006). Simulação de cenários e a estratégia nos negócios. Revista Científica do TECAP: CAP Accounting and Management, 1(1), 3744.

Rojo, C. A.; Brandalise, L. T. (Orgs.) (2010). MBA Gestão Estratégica II (vol. 1, pp. 1-258). Cascavel: Assoeste, v. 1. 258p.

Souza, A. F. D., \& Rojo, C. A. (2010). Análise de investimentos por simulação de cenários baseada em variáveis críticas qualitativas compiladas com Lógica Fuzzy. Revista Científica do TECAP: CAP Accounting and Management, 4(4), 118-126.

Schwartz, P. (1991) The art of the long new. New York: Doubleday/Currency.

Tadeu, H. F. B., \& Silva, J. T. M. (2013). Simulação de cenários para o planejamento estratégico empresarial. Caderno de Ideias FDC, CI 1304.

Teixeira, R. N. C. (2005). Desenvolvimento de um modelo para o planejamento de investimentos em flexibilidade de manufatura em situações de mudanças estratégicas da organização. Tese de Doutorado, Universidade Federal de Santa Catarina: Florianópolis: SC, Brasil.

Wasserman, S., \& Faust, K. (1994). Social network analysis: methods and applications (vol. 8). Cambridge: Cambridge University Press. 